\title{
A Diagnostic Evaluation of Tablet Serious Games for the Assessment of Autism Spectrum Disorder in Young Children
}

Lindsay Millar ${ }^{1,6}$, Alex McConnachie ${ }^{2}$, Helen Minnis ${ }^{3}$, Philip Wilson ${ }^{4}$, Lucy Thompson $^{4,7}$,

Anna Anzulewicz ${ }^{5}$, Krzysztof Sobota ${ }^{5}$, Philip Rowe ${ }^{6}$, Christopher Gillberg ${ }^{7}$, Jonathan

Delafield-Butt ${ }^{1}$

1. Laboratory for Innovation in Autism, University of Strathclyde, Scotland, UK

2. Robertson Centre for Biostatistics, Institute of Health and Wellbeing, University of Glasgow, Scotland, UK

3. Institute of Health \& Wellbeing, University of Glasgow, Scotland, UK

4. Centre for Rural Health, University of Aberdeen, Inverness, Scotland, UK

5. Harimata sp. z o.o., Kraków, Poland

6. Department of Biomedical Engineering, University of Strathclyde, Scotland, UK

7. Gillberg Neuropsychiatry Centre, University of Gothenburg, Sweden

Corresponding author:

Lindsay Millar

1.clarke@strath.ac.uk

Laboratory for Innovation in Autism

GH 850 Graham Hills Building

University of Strathclyde

Glasgow

G1 1XQ

Scotland

UK

Word count: 2,768 


\section{ABSTRACT \\ Introduction}

Recent evidence suggests an underlying movement disruption may be a core component of Autism Spectrum Disorder (ASD) and a new, accessible early biomarker. Mobile smart technologies such as iPads contain inertial movement and touch-screen sensors capable of recording sub second movement patterns during gameplay. A previous pilot study employed machine learning analysis of motor patterns recorded from children 3-5 years old. It identified those with ASD from age- and gender-matched controls with 93\% accuracy, presenting an attractive assessment method suitable for use in the home, clinic or classroom.

\section{Methods and Analysis}

This is a Phase III prospective, diagnostic classification study designed according to the Standards for Reporting Diagnostic Accuracy Studies (STARD) guidelines. Three cohorts are investigated: children developing typically (TD); children with a clinical diagnosis of ASD; and children with a diagnosis of another neurodevelopmental disorder (OND) that is not ASD. The study will be completed in Glasgow, U.K., and Gothenburg, Sweden. The recruitment target is 760 children (280 TD, 280 ASD and 200 OND). Children play two games on the iPad then a third party data acquisition and analysis algorithm (Play.Care, Harimata sp. z o.o., Poland) will classify the data as positively or negatively associated with ASD. The results are blind until data collection is complete, when the algorithm's classification will be compared against medical diagnosis. Furthermore, parents of participants will complete three questionnaires: Strengths and Difficulties Questionnaire; ESSENCE Questionnaire; and the Adaptive Behavioural Assessment System. The primary outcome measure is sensitivity and specificity of Play.Care to detect ASD. Secondary outcomes include the ability of Play.Care to differentiate ASD from OND. 


\section{Ethics and Dissemination}

This study was approved by the Strathclyde University Ethics Committee and the West of Scotland Research Ethics Service Committee 3. Results will be disseminated at international scientific conferences and in peer reviewed publications.

\section{Trial registration}

ClinicalTrials.gov ID: NCT03438994

\section{Keywords}

Autism, diagnosis, digital health, machine learning, motor control, smart technology

\section{ARTICLE SUMMARY}

\section{Strengths and limitations of this study}

- This is the first diagnostic study to evaluate the accuracy of a new iPad game based assessment for Autism Spectrum Disorder in pre-school aged children

- Novel methods are used to collect accurate and reliable movement data as children play

- Children with autism are compared to typically developing controls and children with other neurodevelopmental disorders which are not autism

- This study offers the possibility for a new, non-subjective assessment method for autism 


\section{INTRODUCTION}

Autism spectrum disorder (ASD) is a childhood neurodevelopmental disorder with a prevalence estimated as high as 1 in 59 children in the United States [1]. In the UK, ca. 700,000 individuals live with autism [2] and the aggregate annual cost of healthcare and support is $£ 27.5$ billion [3]. Early identification and consequent early therapeutic intervention may be valuable [4,5]: this may afford family and caregivers opportunity to adjust, and can trigger early healthcare intervention and children's services support. Such provision can produce significant, lifelong health and economic benefit, e.g. [6,7].

Early diagnosis of children with autism remains complex and can be difficult to obtain. Diagnosis currently relies on specialist medical expertise with diagnostic instrumentation dependent on subjectively rated scores during child observations, parent interviews, and testing. These instruments are time consuming, clinically demanding and poorly validated against population controls. Medical diagnosis can be withheld for many years due to waitlist times or uncertainty in clinical diagnostic fit.

Recent identification of motor disturbance in young children who develop ASD presents a new target for early assessment [8]. ASD is typically considered a social and emotional disorder. Therefore, current diagnostic instruments directly address social and emotional aspects of the syndrome. However, motor control underpins social engagement, emotional expression, and cognitive development [9-12], and is accessible to non-invasive, ecologically valid assessment methods from the neonatal stage onward [13].

Children with ASD exhibit a clear deficit in movement, such as delays in the attainment of motor milestones [14], poor coordination [15,16], unusual gait patterns [17-20] and difficulties in gross and fine motor skills [21]. In a meta-analysis of all evidence, Fournier et al [22] concluded motor disruption to be a core feature of autism spectrum disorder. This 
motor perspective is beginning to gain significant clinical and research interest [23] and although movement differences in autism have not yet entered into the diagnostic criteria, the DSM-V now includes 'awkward or clumsy gait' as an associated symptom. More work is required to characterize and determine how these motor differences manifest across the spectrum. The motor signs identified in autism require differentiation from those that extend across the wider spectrum of early symptomatic syndromes eliciting neuropsychiatric clinical examination (ESSENCE; [24]).

Recently, technological developments have miniaturised inertial movement unit sensors that now include tri-axial accelerometers, gyroscopes and magnetometers, together with touchsensitive screens integrated into mobile consumer smart devices such as phones, tablets, and wearable devices such as wristbands and watches. These new devices provide unprecedented access to motor information with high levels of accuracy and reliability, previously restricted to high-end research or clinical laboratories.

In previous work, Anzulewicz, Sobota and Delafield-Butt [25] tested a novel, serious smart tablet game for early identification of young children with autism. Two attractive cartoon games were engineered with bespoke code to collect sensor data (inertial and touch screen sensors) as the children engaged for five minutes with each game. Machine learning analysis identified those children diagnosed with autism with 93\% accuracy, proving the principle that smart game identification of autism in children was a viable solution for ecological, accessible assessment. Furthermore, this assessment methodology required no professional training to deploy, no verbal instructions were required to engage the child, and only limited supervision was necessary. The result of the assessment is purely statistical; no subjective assessment is required. Thus, we propose this serious game digital health tool could offer an attractive addition to the diagnostician's tool box or serve as a paediatric public health screening tool. 
The current best validated sub-component of the gold-standard in autism diagnosis, the Autism Diagnostic Observation Schedule (ADOS) Version 2, yields diagnostic performance of, at most, $91 \%$ sensitivity and $71 \%$ specificity, or $82 \%$ sensitivity and $88 \%$ specificity when the algorithm is adjusted to increase specificity [26]. The iPad serious game assessment, now called Play.Care, demonstrated a sensitivity and specificity of $83 \%$ and $85 \%$, respectively [25]. Serious game assessment by smart device appears to be an attractive resource that could improve sensitivity in autism diagnosis.

The aim of this study is to validate the predictive value of the Play.Care assessment for autism diagnosis by conducting a multi-site, controlled diagnostic study. Further aims include assessing the ability of Play.Care to differentiate ASD from other childhood developmental disorders. The primary outcome measures will be sensitivity and specificity of Play.Care to detect ASD in the general population.

Limitations inherent in the earlier pilot study are overcome in the diagnostic trial presented here. The pilot study was based on a small sample of 82 children recruited from a small number of specialist day-care centres in Poland. Such limited subject recruitment is prone to selection bias and site-specific effects and precludes assessment of predictive value in population-based settings. Furthermore, the pilot study employed the same dataset to train and test the machine learning algorithm. It did so with 10 repetitions of a 10 -fold crossvalidation procedure that is not prone to over-fitting. Nevertheless, it only trained and tested patterns on that particular dataset. To alleviate both concerns, this study sets out to test the algorithm previously developed and published [25] on the new, blinded data obtained independently of the Play.Care software engineers in an international, multisite diagnostic evaluation with a general population cohort and Phase III clinical study structure and size. This trial is designed to test the Play.Care assessment to determine its generalized, real-world prediction rates across the range of possible ASD expression, as well as against TD and 
OND variability. Should the sensitivity and specificity of the assessment fall within clinically useful levels comparable to or exceeding current observer-rated tests (ADOS-2, Autism Diagnostic Interview - Revised; ADI-R), we expect this instrument to be an attractive and useful addition to the diagnostic tool box, accessible to a wide range of professionals in psychiatry, paediatrics and specialist children's services, including those in education. This solution is attractive and timely, and benefits from the surge in machine learning analysis of smart tech device data. In our case, this is a fun, useful and mobile serious game for autism assessment.

\section{METHODS AND ANALYSIS}

\section{Study Design}

This study is a Phase III prospective, diagnostic classification study. The protocol follows the Standards for Reporting Diagnostic accuracy studies (STARD) guidelines [27]. The study will be a multi-centre, international trial based in Glasgow, Scotland, UK and Gothenburg, Sweden. The Glasgow cohort will comprise a clinical sample and the Gothenburg cohort will comprise a general population sample. The study will have three groups of participants: children who have received a clinical diagnosis of ASD; children who have received a clinical diagnosis of an Other Neurodevelopmental Disorder (OND; see

Table 2 for Inclusion/Exclusion criteria); and children developing typically (TD).

\section{Participants}

A total of 760 children aged thirty months to five years 11 months will be recruited. Table 1 illustrates the participant numbers between sites and groups and Table 2 details inclusion/exclusion criteria for each group.

Table 1. Participant numbers across groups and sites

\begin{tabular}{lccc|l}
\hline Site & ASD & OND & TD & Total \\
\hline Glasgow & 100 & 140 & 180 & 420 \\
\hline Gothenburg & 180 & 60 & 100 & 340 \\
\hline Total & 280 & 200 & 280 & $\mathbf{7 6 0}$ \\
\hline
\end{tabular}


Table 2. Participant inclusion and exclusion criteria

\begin{tabular}{|c|c|c|}
\hline Group & Inclusion criteria & Exclusion criteria \\
\hline All participants & $\begin{array}{l}\text { Aged } 30 \text { months to five years } 11 \\
\text { months }\end{array}$ & \\
\hline \multirow[t]{2}{*}{$\begin{array}{l}\text { Participants with a } \\
\text { diagnosis of ASD }\end{array}$} & $\begin{array}{l}\text { Diagnosis of autism spectrum } \\
\text { disorder on the basis of DSM-V } \\
\text { criteria or equivalent ICD-10 } \\
\text { framework }\end{array}$ & $\begin{array}{l}\text { Uncorrected sensory (visual, } \\
\text { hearing) impairments }\end{array}$ \\
\hline & & $\begin{array}{l}\text { Presence of any motor } \\
\text { impairments or behavioural } \\
\text { impairment that may obstruct } \\
\text { testing }\end{array}$ \\
\hline \multirow[t]{2}{*}{$\begin{array}{l}\text { Participants with a } \\
\text { diagnosis of OND }\end{array}$} & $\begin{array}{l}\text { Other childhood developmental } \\
\text { disorders including, but not limited } \\
\text { to Intellectual Disability, Non- } \\
\text { verbal Disability, Communication } \\
\text { Disability, Attention Deficit } \\
\text { Hyperactivity Disorder and } \\
\text { Developmental Coordination } \\
\text { Disorder, Down's Syndrome and } \\
\text { Cerebral Palsy. }\end{array}$ & $\begin{array}{l}\text { Sub clinical or secondary } \\
\text { expressions of ASD }\end{array}$ \\
\hline & & $\begin{array}{l}\text { Uncorrected hearing or vision } \\
\text { impairments }\end{array}$ \\
\hline TD participants & Within age range & $\begin{array}{l}\text { Diagnosis or suspicion of } \\
\text { - Neuropathology } \\
\text { - Psychopathology } \\
\text { - Sensory or motor } \\
\text { disorder including mild } \\
\text { tremors }\end{array}$ \\
\hline
\end{tabular}

Group sizes were decided upon by assuming Play.Care will achieve a moderate $85 \%$ sensitivity. In this case, a study with 200 children with ASD will yield a confidence interval with a width of $\pm 4.9 \%$ i.e. the final sensitivity calculation will be accurate to within this range. The target recruitment size for this group is 280 children to afford some reduction in sensitivity or a significant non-completion rate while preserving a narrow confidence interval. The wider population recruited in this study may reduce sensitivity, and inclusion of low 
functioning children with ASD increases the likelihood of disengagement with, or disinterest in the Play.Care assessment. Assuming a similar level of specificity, the same precision will be obtained with 200 TD children. Furthermore, if the sensitivity (or specificity) is as high as $90 \%$, there will be $80 \%$ power to show that the sensitivity (or specificity) exceeds $83.0 \%$, and $90 \%$ power to show it exceeds $81.9 \%$. On the other hand, if the sensitivity (or specificity) is only $70 \%$, there will be $91 \%$ power to show that it is below $80 \%$. That is, if the Play.Care assessment is under performing, which is the major concern of healthcare services, this study has a high probability of detecting it.

For the Glasgow cohort, recruitment of children with ASD or OND will be facilitated through the NHS Greater Glasgow and Clyde Specialist Children's Services, namely the Southbank Child Development Centre, as well as specialist children's centres, nurseries, and schools. ASD and OND recruitment will occur via two streams. The first will recruit children who receive a positive diagnosis of ASD or OND following an assessment at the Southbank Centre. The second will recruit children already diagnosed with ASD or OND from specialist nurseries, preschools and development centres. Typically developing children will be recruited from local private nurseries in the Greater Glasgow area.

The Gothenburg cohort is a general population sample as all children are screened for ASD within the Gothenburg area at 30 months. Families of children screened positively for ASD are referred to the Gillberg Neuropsychiatry Centre (GNC) in Gothenburg for neuropsychiatric assessment and it is at this stage that families will be made aware of the study and asked if they would like to participate. OND children in Gothenburg will be recruited through Speech and Language Therapists in collaboration with the GNC.

\section{Diagnostic Device}

The diagnostic tool under assessment in this study is the Play.Care iPad mini application, developed by Harimata sp. z o.o., Poland. The assessment consists of two games played on 
the iPad. The first game is called 'Sharing' and requires the child to tap a piece of food to split it into four pieces, available for sharing with the four game characters waiting patiently (Figure 1a). The second game is called 'Creativity' and requires the child to trace the outline of a drawing and then colour it in (Figure 1b). Each game consists of a two minute training phase where the researcher and/or parent can assist the child, and a five minute assessment phase where the child must play unassisted. Following completion of the assessment, no further participation is required. Anzulewicz et al. [25] details the data analytics employed, including identification of movement patterns by calculation of more than two hundred features that are assessed by an algorithm that statistically determines the diagnostic prediction.
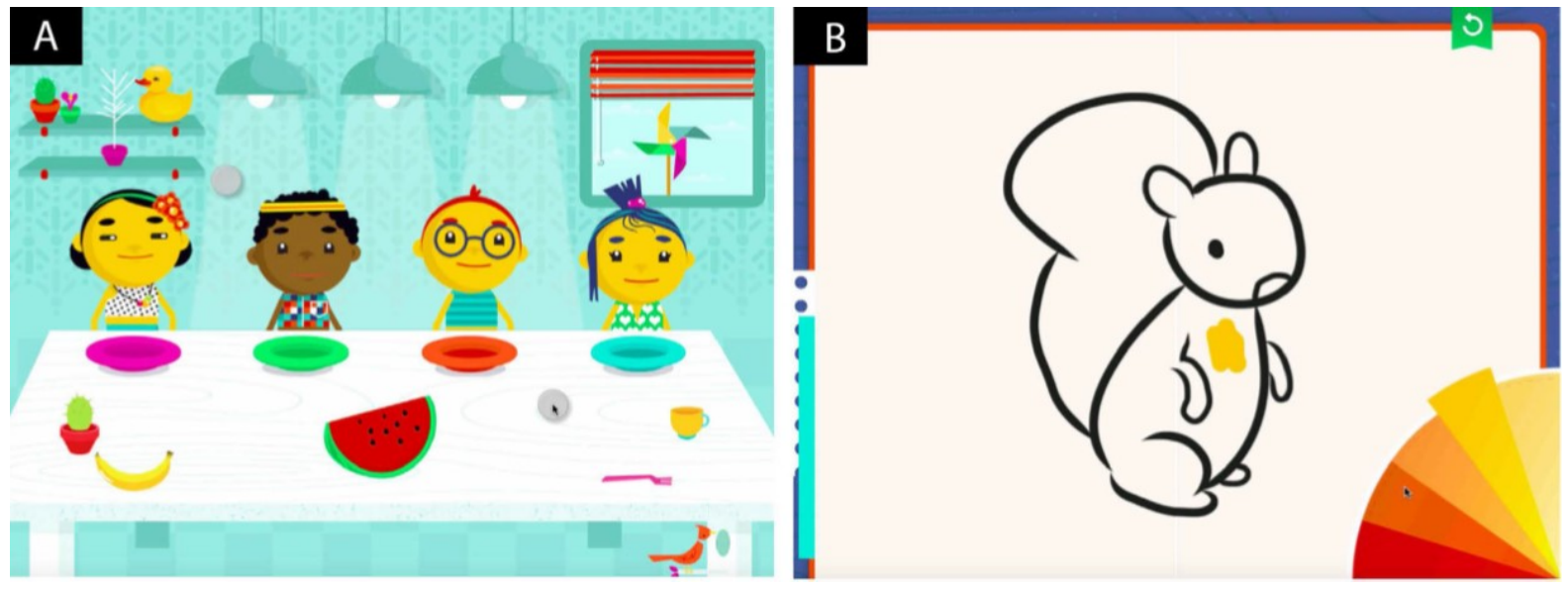

Figure 1. Play.Care assessment games. (a) 'Sharing'. The child must tap the fruit to divide it into four and share it amongst game characters. When all four characters have a slice of fruit, they express happiness for three seconds before the fruit is replaced with another food and the characters return to their neutral position. (b) 'Creativity'. The child is free to choose an object or animal which they must trace and then colour in freely by choosing a colour form the colour wheel. If the child is satisfied, a new shape can be chosen at any time [25].

The iPads used in this study are commercially available Apple products currently widely used for leisure, business and research. The iPad specifications employed here are as follows: white bevel, silver back, non-cellular iPad mini 4, 128GB. The iOS version will be the latest version available upon purchase of the iPads. These specifications will remain fixed for the duration of the study. Furthermore, software updates will be switched off to prevent 
automatic updates of iOS which may alter the sampling rate of the internal gyroscope and accelerometers.

\section{Procedure}

Potential participants in the ASD and OND assessment stream will first be approached by the clinician undertaking the assessment. The family will be presented with the Patient Information Sheet (PIS) following a positive diagnosis and if the clinician judges them to be suitable participants. The family will complete an interest slip indicating they are willing to be further contacted by the research team regarding the study. This method was adopted to allow participation to be strictly 'opt-in' and to prevent the research team contacting families who had not expressed an interest in participating. After a minimum period of 24 hours, the research team will contact interested families to ask if they would like to participate and to arrange a data collection appointment.

Potential participants in the ASD and OND already diagnosed stream will be first approached at their child's preschool where the research team will introduce the study during a parent's evening or similar event. At this point, the PIS will be made available and families who are interested will complete an interest slip, as above, and after a minimum of 24 hours the research team will contact them.

Potential participants who are developing typically will be recruited through local private nurseries. Nursery staff will circulate the PIS amongst parents and those who wish their child to participate will return a completed consent form to the nursery. Prior to completing the Play.Care assessment, informed consent will be given by the child's parents or caregivers. The researcher will then complete a Case Report Form (CRF) for participants in the ASD and OND groups which details study ID, Scottish Community Health Index (“CHI”) number, name, address, postcode, phone number and general practitioner (GP) details. Participants' $\mathrm{CHI}$ numbers will be recorded to allow follow up for up to 10 years following participation. 
This will provide potential future insight into whether diagnoses remain consistent into adolescence and whether any other diagnoses are received. Any further diagnoses can then be compared to the iPad data to determine any correlations between new diagnoses and movement characteristics in childhood.

An electronic CRF (eCRF) will be completed detailing the child's emotional state on the day of data collection and a number of additional psychometric assessments including the Strengths and Difficulties Questionnaire (SDQ), the Early Symptomatic Syndromes Eliciting Neurodevelopmental Clinical Examinations Questionnaire (ESSENCE-Q) and the Adaptive Behaviour Assessment System (ABAS) at Glasgow and the Vinelands Adaptive Behaviour Scale (VABS) in Gothenburg. In some cases, additional data will be recorded from instruments employed as part of the child's neuropsychiatric assessment: Wechsler Preschool and Primary Scale of Intelligence (WPPSI); Autism Diagnostic Interview Revised (ADI-R); and Autism Diagnostic Observation Schedule 2 (ADOS-2). The diagnostic criteria that determine a child's diagnosis (ICD 10 or DSM V) will be recorded. For participants in the ASD and OND groups, psychometric questionnaires will be completed at an appropriate time during or following the data collection appointment.

Following completion of the CRF and eCRF, the child will complete the Play.Care assessment. During the assessment, the iPad is protected within a spongy back-cover and bumper (iPad Mini2 Air Protect, Belkin, U.S.A.) and is placed flat on the table top, where it will remain for the duration of the assessment. Parents/caregivers will have the option to consent to their child being videotaped during the assessment. If consent is given, a video camera will be setup and recording will begin prior to the child starting the assessment. If a participant is unable to complete the assessment (e.g. they become distracted or distressed), eCRF and CRF data will still be recorded and the participant will remain a subject in the study. This group will be classified as "Play.Care incompletes" and will 
provide insight into the clinical applicability of the assessment. This group will be monitored at monthly intervals. If the rate of Play.Care incompletes reaches a level where it will impact the statistical power of the study, administration protocols will be reviewed and adjusted to ensure best possible compliance. This criterion will apply to the Glasgow cohort of participants only. The Gothenburg cohort is a general population sample and therefore participation cannot be controlled dependent on failure rates. If a participant does not have a confirmed clinical diagnosis by the end of the study, they will fall into the category of “diagnosis failures". The acceptable level of diagnosis failures is $7.5 \%$, or, up to 21 subjects in the ASD group and 15 in the OND group.

Throughout the data collection phase, all members of the research team, the trial consortium and Harimata will be blinded to the Play.Care diagnostic prediction until all data have been collected. Following data collection, clinical diagnoses will be compared with Play.Care diagnostic predictions. The primary outcome measure is the ability of Play.Care to identify ASD cases within the general population (Gothenburg) and clinic (Glasgow) by assessing classification efficacy against TD children. The secondary outcome measures are the ability of Play.Care to differentiate these ASD children from OND children and to correlate movement data to adaptive behaviour scores.

\section{Patient and Public Involvement}

The public have not been involved in the development of the study protocol. Patients will be involved as study participants and a lay summary of the results will be made available to families who participated.

\section{ETHICS AND DISSEMINATION}

This study was granted approval by the Strathclyde University Ethics Committee and the West of Scotland Research Ethics Service Committee 3 (reference number: 17-WS-0223 231435). Following completion of data collection, results will be disseminated internally at 
Strathclyde University via the Laboratory for Innovation in Autism website, via a final report to Harimata sp. z o.o., at national and international scientific conferences and in peer reviewed scientific journals. A lay summary of study results will also be provided for participating families.

\section{REFERENCES}

1 Baio J, Wiggins L, Christensen D, et al. Prevalence of Autism Spectrum Disorder Among Children Aged 8 Years - Autism and Developmental Disabilities Monitoring Network, 11 Sites, United States, 2014. MMWR Suveill Summ 2018;67:1-23.

2 Brugha TS, McManus S, Bankart J, et al. Epidemiology of autism spectrum disorders in adults in the community in England. Arch Gen Psychiatry 2011;68:459-65. doi:10.1001/archgenpsychiatry.2011.38

3 Järbrink K, Knapp M. The Economic Impact of Autism in Britain. Autism 2001;5:7-22. doi:10.1177/1362361301005001002

4 Bradshaw J jbradshaw@educationucsb ed, Steiner A, Gengoux G, et al. Feasibility and Effectiveness of Very Early Intervention for Infants At-Risk for Autism Spectrum Disorder: A Systematic Review. Journal of Autism \& Developmental Disorders 2015;45:778-94. doi:10.1007/s10803-014-2235-2

5 Pickles A, Le Couteur A, Leadbitter K, et al. Parent-mediated social communication therapy for young children with autism (PACT): long-term follow-up of a randomised controlled trial. The Lancet 2016;388:2501-9. doi:10.1016/S0140-6736(16)31229-6

6 Peters-Scheffer N, Didden R, Korzilius H, et al. Cost comparison of early intensive behavioral intervention and treatment as usual for children with autism spectrum disorder in the Netherlands. Research in Developmental Disabilities 2012;33:1763-72. doi:10.1016/j.ridd.2012.04.006

7 Chasson GS, Harris GE, Neely WJ. Cost Comparison of Early Intensive Behavioral Intervention and Special Education for Children with Autism. J Child Fam Stud 2007;16:401-13. doi:10.1007/s10826-006-9094-1

8 Trevarthen C, Delafield-Butt JT. Autism as a developmental disorder in intentional movement and affective engagement. Front Integr Neurosci 2013;7. doi:10.3389/fnint.2013.00049

9 Anzulewicz A, Sobota K, Delafield-Butt JT. Toward the Autism Motor Signature: Gesture patterns during smart tablet gameplay identify children with autism. Scientific Reports 2016;6:31107. doi:10.1038/srep31107

10 Adolph KE, Tamis-Lemonda CS, Karasik LB. Cinderella indeed - a commentary on Iverson's 'Developing language in a developing body: the relationship between motor development and language development' $<\mathrm{a}$ href $=" \# \mathrm{fn} 01 \mathrm{a} "{ }^{*}</ \mathrm{a}>$. Journal of Child Language 2010;37:269-73. doi:10.1017/S030500090999047X 
11 Trevarthen C, Delafield-Butt J. Biology of Shared Meaning and Language Development: Regulating the Life of Narratives. In: The Infant Mind: Origins of the Social Brain. New York: : Guilford Press 2013. 167-99.

12 Trevarthen C, Delafield-Butt J. Development of consciousness. In: The Cambridge Encyclopedia of Child Development. University Press 2017. 821-35.

doi:10.1017/9781316216491.131

13 Delafield-Butt JT, Freer Y, Perkins J, et al. Prospective organization of neonatal arm movements: A motor foundation of embodied agency, disrupted in premature birth. Developmental Science;0:e12693. doi:10.1111/desc.12693

14 Teitelbaum P, Teitelbaum O, Nye J, et al. Movement analysis in infancy may be useful for early diagnosis of autism. PNAS 1998;95:13982-7. doi:10.1073/pnas.95.23.13982

15 Ghaziuddin M, Butler E. Clumsiness in autism and Asperger syndrome: a further report. J Intellect Disabil Res 1998;42 ( Pt 1):43-8.

16 Mari M, Castiello U, Marks D, et al. The reach-to-grasp movement in children with autism spectrum disorder. Philosophical Transactions of the Royal Society of London B: Biological Sciences 2003;358:393-403. doi:10.1098/rstb.2002.1205

17 Fabbri-Destro M, Cattaneo L, Boria S, et al. Planning actions in autism. Exp Brain Res 2009;192:521-5. doi:10.1007/s00221-008-1578-3

18 Matson JL, Mahan S, Kozlowski AM, et al. Developmental milestones in toddlers with autistic disorder, pervasive developmental disorder--not otherwise specified and atypical development. Dev Neurorehabil 2010;13:239-47. doi:10.3109/17518423.2010.481299

19 Ming X, Brimacombe M, Wagner GC. Prevalence of motor impairment in autism spectrum disorders. Brain and Development 2007;29:565-70.

doi:10.1016/j.braindev.2007.03.002

20 Schmitz C, Martineau J, Barthélémy C, et al. Motor control and children with autism: deficit of anticipatory function? Neuroscience Letters 2003;348:17-20. doi:10.1016/S0304-3940(03)00644-X

21 Lloyd M, MacDonald M, Lord C. Motor skills of toddlers with autism spectrum disorders. Autism 2013;17:133-46. doi:10.1177/1362361311402230

22 Fournier KA, Hass CJ, Naik SK, et al. Motor Coordination in Autism Spectrum Disorders: A Synthesis and Meta-Analysis. Journal of Autism \& Developmental Disorders 2010;40:1227-40. doi:10.1007/s10803-010-0981-3

23 Torres EB, Donnellan AM. Autism: The Movement Perspective. Frontiers Media SA 2015.

24 Hatakenaka Y, Kotani H, Yasumitsu-Lovell K, et al. Infant Motor Delay and Early Symptomatic Syndromes Eliciting Neurodevelopmental Clinical Examinations in Japan. Pediatric Neurology 2016;54:55-63. doi:10.1016/j.pediatrneurol.2015.09.008 
25 Anzulewicz A, Sobota K, Delafield-Butt JT. Toward the Autism Motor Signature: Gesture patterns during smart tablet gameplay identify children with autism. Scientific Reports 2016;6:31107. doi:10.1038/srep31107

26 de Bildt A, Sytema S, van Lang NDJ, et al. Evaluation of the ADOS Revised Algorithm: The Applicability in 558 Dutch Children and Adolescents. $J$ Autism Dev Disord 2009;39:1350-8. doi:10.1007/s10803-009-0749-9

27 Bossuyt PM, Reitsma JB, Bruns DE, et al. STARD 2015: an updated list of essential items for reporting diagnostic accuracy studies. BMJ 2015;351:h5527.

doi:10.1136/bmj.h5527

\section{AUTHOR STATEMENT}

JDB, CG, HM, PR, PW, LT, AM, KS, and AA devised the study design. LM, JDB, CG, HM, PR, AM, PW, and LT developed the study protocol with input from KS and AA. Sample size calculations were carried out by AM. KS, AA \& JDB were responsible for technical development and pilot work. This paper was written by LM \& JDB with input from all coauthors.

\section{DATA STATEMENT}

Data obtained in this trial will be held by the University of Strathclyde and the funder for use within the trial research consortium (authors) for a period of up to 10 years. All scientific results will be published in the peer-reviewed literature. The funder retains the right to a 2year embargo on such publication.

\section{CONFLICTS OF INTEREST}

The academic authors LM, HM, PW, AM, PR, CG, and JDB are members of the Trial Steering and Management Committees and declare no financial interest in this product or the funding company, Harimata sp. z o.o. Co-authors AA and KS are board members of the Harimata sp. z o.o that intends to commercialise the Play.Care assessment technology. AA and KS have options vesting in the company. AA is a voting member of the Trial Steering Committee. 


\section{FUNDING}

This work was subcontracted to the University of Strathclyde by Harimata sp. z o.o. as part of a Horizon 2020 SME instrument, grant number 756079. 